\title{
Effect of Cardiac Stem Cells in Patients with Ischemic Cardiomyopathy: Initial Results of the SCIPIO Trial
}

\author{
Roberto Bolli ${ }^{1}$, Atul R. Chugh ${ }^{1}$, Domenico D'Amario², John H. Loughran', Marcus F. \\ Stoddard ${ }^{1}$, Sohail Ikram ${ }^{1}$, Garth M. Beache ${ }^{3}$, Stephen G. Wagner ${ }^{1}$, Annarosa Leri' ${ }^{2}$, Toru \\ Hosoda $^{2}$, Julius B. Elmore ${ }^{1}$, Polina Goihberg ${ }^{2}$, Donato Cappetta ${ }^{2}$, Naresh K. Solankhi ${ }^{1}$, \\ Ibrahim Fahsah ${ }^{1}$, D. Gregg Rokosh ${ }^{1}$, Mark S. Slaughter ${ }^{4}$, Jan Kajstura ${ }^{2}$, and Piero Anversa ${ }^{2}$ \\ ${ }^{1}$ Division of Cardiovascular Medicine, University of Louisville, Louisville, KY \\ ${ }^{2}$ Departments of Anesthesia and Medicine and Division of Cardiovascular Medicine, Brigham and \\ Women's Hospital, Harvard Medical School, Boston, MA \\ ${ }^{3}$ Department of Radiology, University of Louisville, Louisville, KY \\ ${ }^{4}$ Division of Cardiothoracic Surgery, University of Louisville, Louisville, KY
}

\section{Summary}

Background-C- $-\mathrm{kit}^{+}$lineage ${ }^{-}$cardiac stem cells (CSCs) improve postinfarction left ventricular (LV) dysfunction in animals; however, their efficacy in humans is unknown.

Methods-In February 2009, we began SCIPIO ( $\underline{\text { Stem }}$ Cell $\underline{\text { Infusion in }}$ Patients with $\underline{\text { Ischemic }}$ Cardiomyopathy), a Phase I, randomized, open-label trial of CSCs in patients with postinfarction LV dysfunction (ejection fraction $[\mathrm{EF}] \leq 40 \%$ ) who underwent coronary bypass surgery.

Autologous CSCs were isolated from the right atrial appendage and re-infused intracoronarily $4 \pm$ 1 months after surgery; controls received no treatment. In Stage A, 9 treated and 4 control patients were consecutively enrolled to assess the feasibility and short-term safety of CSCs. Then, in Stage $\mathrm{B}$, patients were randomized to the treated or control arm in a 2:3 ratio using a block randomization scheme and a block size of five. Primary (safety) and secondary (efficacy) endpoints were assessed at serial times after enrollment.

Findings-Autologous CSCs were successfully isolated and expanded in 80 out of 81 patients. In 16 treated patients, no CSC-related adverse effects have been observed. LVEF (3D

\footnotetext{
Address for Correspondence: Roberto Bolli, M.D., Division of Cardiovascular Medicine, 550 S Jackson Street, ACB Bldg, $3^{\text {rd }}$ Floor, Louisville, KY 40202, (502) 852-1837, rbolli@ louisville.edu.

Contributors. RB was the chief investigator for the study and designed and managed the study with input from the group. PA, DD, AL, TH, PG, DC, DGR, and JK designed, performed, and interpreted all experiments involving CSCs. MS and SW designed, performed, and interpreted all echocardiographic studies. GB designed, interpreted, and performed all cMR studies. SI designed and performed all interventional procedures. ARC, JHL, JBE, NKS, IF, and MSS contributed to the design of the study, collection and interpretation of data, and preparation of the manuscript. All authors participated in data interpretation. RB drafted the first and subsequent versions of this report with input and key revisions by all authors, who reviewed and approved the final submitted report. Conflicts of Interest. PA is a member of Autologous, LLC. The other authors declare that they have no conflicts of interest. Government Trial Number: NCT00474461

Publisher's Disclaimer: This is a PDF file of an unedited manuscript that has been accepted for publication. As a service to our customers we are providing this early version of the manuscript. The manuscript will undergo copyediting, typesetting, and review of the resulting proof before it is published in its final citable form. Please note that during the production process errors may be discovered which could affect the content, and all legal disclaimers that apply to the journal pertain.
} 
echocardiography) increased from $30.3 \pm 1.9 \%$ before CSC infusion to $38.5 \pm 2.8 \%$ at 4 months after infusion, $(P=0.001, \mathrm{n}=14)$. This was associated with an improvement in regional wall motion score index (echocardiography) ( $1.91 \pm 0.09$ vs. $1.73 \pm 0.09, P=0.005)$, NYHA functional class $(2.19 \pm 0.16$ vs. $1.63 \pm 0.16, P=0.003)$, and quality of life (MLHFQ score, $46.44 \pm 5.22$ vs. 26.69 $\pm 4.92, P<0.0001)$. In contrast, in 7 control patients, none of these variables changed appreciably during the corresponding time-interval. Importantly, the salubrious effects of CSCs were even more pronounced at 1 year (e.g., LVEF increased by $12.3 \pm 2.1 \%$ vs. pre-CSCs, $P=0.0007, \mathrm{n}=8$ ), suggesting that CSCs continue to improve LV function beyond the first 4 months. In the 7 treated patients in whom cardiac magnetic resonance (cMR) imaging could be performed, infarct size decreased by $7.8 \pm 1.7 \mathrm{~g}(23.8 \%)$ at 4 months $(P=0.004)$ and $9.8 \pm 3.5 \mathrm{~g}(30.3 \%)$ at 1 year $(P=0.04)$.

Interpretation-These initial results in humans are very encouraging, and suggest that infusion of autologous CSCs is effective in improving LV systolic function and reducing infarct size in patients with heart failure.

\section{Introduction}

Heart failure (HF) is a common, lethal, disabling, and expensive condition. Its prevalence in industrialized nations has reached epidemic proportions (e.g., $\sim 1$ million cases in the $\mathrm{UK}^{1}$ and nearly 6 million in the US ${ }^{2}$ ), and continues to rise. Despite advances over the last 30 years, the prognosis of patients who are hospitalized with HF remains poor, with a 5-year mortality that approaches $50 \%^{2}$ - worse than that of patients with breast or colon cancer ${ }^{3}$. The most common cause of HF in the Western world is ischemic heart disease (IHD) ${ }^{2}$. Therefore, HF secondary to IHD constitutes a major public health problem worldwide but do not address the fundamental problem, i.e., the loss of cardiac tissue. Consequently, there has been increasing interest in attempting to repair the failing heart with the use of stem cells, since this approach has the potential to regenerate dead myocardium and thus alleviate the underlying cause of $\mathrm{HF}^{4}$.

The adult heart contains a population of cardiac stem cells (CSCs) that are identified by the expression of the surface receptor tyrosine kinase c-kit ${ }^{5-7}$. These cells are self-renewing, clonogenic, and multipotent, i.e., they differentiate into all three major cardiac lineages (myocytes, vascular smooth muscle cells, and endothelial cells) ${ }^{5,7-11}$. Numerous studies have demonstrated that transplantation of CSCs in animal models of post-myocardial infarction (MI) HF limits left ventricular (LV) remodeling and improves LV function, in the setting of both an acute and a chronic MI ${ }^{5,7-11}$. Despite these encouraging preclinical results, however, the effects of CSCs in patients have not been explored.

We therefore undertook a Phase I clinical trial of CSCs. Here, we report the initial results obtained in the first 16 CSC-treated and 7 control patients.

\section{Methods}

\section{Study protocol}

The protocol is illustrated in supplemental Fig. 1. SCIPIO was a Phase I, randomized, openlabel, single-center trial of autologous CSCs in patients with severe HF secondary to 
ischemic cardiomyopathy. The target population consisted of patients who underwent coronary artery bypass (CABG) surgery and had LV ejection fraction (EF) $\leq 40 \%$ and an old MI. Enrollment was based on eligibility screening at two time points. Initial screening took place within 2 weeks of CABG surgery (Fig. 1). Inclusion criteria were LVEF $\leq 40 \%$ and evidence of a myocardial scar. Inclusion and exclusion criteria are listed in supplemental Table 1 and can be found online (http://clinicaltrials.gov/ct2/show/NCT00474461). Final screening was performed $4 \pm 1$ months after CABG surgery using the same criteria (Fig. 1). All parameters of cardiac performance obtained at final screening were considered baseline data.

At the time of CABG surgery, the right atrial appendage was harvested at the University of Louisville and shipped to the laboratory of one of the investigators (P.A.) at the Brigham and Women's Hospital, where CSCs were isolated and expanded as described below. The pre-final CSC product was then transported to a GMP laboratory in Louisville for sterility testing, cell count, and determination of viability. After confirmation of negative microbial testing, the final CSC product was prepared in $12 \mathrm{~mL}$ of PlasmaLyte A for infusion at the University of Louisville.

In patients assigned to the treated arm, autologous CSCs were infused intracoronarily at $4 \pm$ 1 months after CABG surgery. An over-the-wire balloon catheter (Boston Scientific Quantum Maverick non-compliant balloon or Abbott Laboratories Voyager RX balloon) was advanced into the proximal coronary artery or graft supplying the infarcted LV region. The balloon was inflated for 3 min using low pressure to stop coronary flow, during which time CSCs were infused distally through the central port of the catheter. Four inflations with 3 min of intervening reflow were performed. The number of CSCs infused depended on the number and location of the infarcts. In patients with one myocardial scar, $1 \times 10^{6}$ cells were infused into anterior wall infarcts and $0.5 \times 10^{6}$ cells into infarcts within the left circumflex or right coronary artery territories. In patients with multiple regions of infarction, $0.5 \times 10^{6}$ cells were infused into two different vascular territories, so as not to exceed a total of $1 \times$ $10^{6}$ cells. Following CSC infusion, patients were monitored during an overnight hospitalization. Control patients did not undergo cardiac catheterization.

In treated patients, 2-D and 3-D transthoracic echocardiograms, routine laboratory tests, physical examination, and NYHA class assessment were performed before CSC infusion and 1, 4, and 12 months thereafter; in addition, routine laboratory tests and physical examination were performed at $24 \mathrm{~h}, 1$ and 2 weeks, and 8 months after CSCs. The Minnesota Living with Heart Failure Questionnaire (MLHFQ) was completed by subjects prior to CSC infusion and 4 and 12 months later. In patients with no contraindications, cardiac magnetic resonance (cMR) imaging was performed before CSCs and 4 and 12 months thereafter. A 24-h ambulatory monitor was used for arrhythmia detection at 1 and 4 weeks after CSC infusion.

The primary goal of the study was to investigate the safety and feasibility of using autologous CSCs for the treatment of HF resulting from IHD. The secondary goal was to garner initial insights into the effects of CSCs on LV function, infarct size, and functional 
status. Since SCIPIO is the first study of CSCs in humans, the results will be important for developing this new form of cell therapy.

\section{Isolation and expansion of CSCs}

The atrial samples were cut into small pieces $\left(<1 \mathrm{~mm}^{3}\right)$ and suspended in 2-5 ml Ham's F12 medium containing 1-3 mg/ml of collagenase NB 6 (Crescent Chemicals Corporation). Following digestion, cells were plated in Petri dishes containing Ham's F12 medium (Cambrex) supplemented with 10\% fetal bovine serum (Hyclone), $100 \mathrm{ng} / \mathrm{ml}$ recombinant human bFGF (PeproTech), $0.2 \mathrm{mM} \mathrm{L-glutathione} \mathrm{(Sigma),} \mathrm{and} 5 \mathrm{mU} / \mathrm{ml}$ human erythropoietin (Sigma). Subsequently, cells were expanded and subjected to immunomagnetic sorting with microbeads (human CD117 MicroBead kit, Miltenyi) to obtain c-kit-positive $\mathrm{CSCs}^{7,12}$. Approximately $2 \times 10^{6} \mathrm{CSCs}$ were obtained per patient.

\section{Characterization of CSCs}

To measure the fraction of ${\mathrm{c}-\mathrm{kit}^{+}}^{+}$lineage ${ }^{-}$cells in the preparation, a small aliquot of CSCs was fixed in $4 \%$ paraformaldehyde and incubated for $45 \mathrm{~min}$ at $37^{\circ} \mathrm{C}$ with a c-kit antibody or a cocktail of primary antibodies recognizing myocytes (GATA 4, Nkx2.5, Mef2c, asarcomeric actin, connexin 43), smooth muscle cells (SMCs; a-smooth muscle actin), and endothelial cells (ECs; von Willebrand factor). FACS analysis was performed with FACSAria (Becton Dickinson) or Accuri C6 (Accuri Cytometers) instruments 5, 7, 12. Quantitative measurements of telomere length were made by Q-FISH and confocal microscopy or by flow-FISH $7,12,13$. The catalytic activity of telomerase was assessed by quantitative PCR ${ }^{12,13}$. To measure population doubling time (PDT), CSCs were plated at low-density and the number of cells was determined daily. PDT was computed by linear regression of $\log _{2}$ values of cell number. To determine the fraction of cells that reached replicative senescence and irreversible growth arrest, cultures were stained for the senescence-associated protein $\mathrm{p} 16^{\mathrm{INK} 4 \mathrm{a}} 7,12,13$. An expanded description of these methods is in the Online Supplement.

\section{Echocardiography}

Full volume real-time three-dimensional echocardiography (3DE) images were obtained from an apical window. The entire LV was included for volumetric measurement by fullvolume 3D data sets acquired by combining 4 ECG gated pyramidal subvolumes. Images were acquired over four cardiac cycles using a matrix array ultrasonographic transducer (X3-1 transducer, Philips Medical Systems, Bothell, WA). Measurements of 3D volumes and EF were performed off-line using a semi automated algorithm by QLAB (Version 3.1, Philips Medical Systems). From an apical full volume acquisition, frames for LVEDV and LVESV measurement were identified. Endocardial contour tracing was performed with a semi-automatic border detection algorithm and manually adjusted if needed as follows: after identifying the apex and mitral annulus on 4-chamber and 2-chamber slices, a preconfigured ellipse was automatically fitted to the endocardial borders of each frame and manually adjusted as required in appropriate planes. LVEDV and LVESV were measured from the 3D volumes, and EF derived. All measurements were performed by an experienced echocardiographer (MFS). Wall motion analysis was performed using the 16-segment model as recommended by the American Society for Echocardiography ${ }^{14}$. 
cMR images were acquired using a 1.5T Espree system. (Siemens Medical Solutions, Erlangen, Germany). Cardiac gated, TrueFISP Cine acquisitions (25 temporal frames) were performed during breath holding, with phased array reception coils. Typical parameters were as follows: TR $50 \mathrm{~ms}$, TE $1.5 \mathrm{~ms}$, Flip Angle: 80 with a spatial resolution of $1.4 \mathrm{~mm} \times 3.1$ $\mathrm{mm}$ in-plane. Default slice thickness was $8 \mathrm{~mm}$, with 10 to 12 short-axis image sections, for complete coverage of the left ventricle. Late gadolinium enhancement for infarct assessment was also done using Multihance (Bracco, Inc., Milan, Italy) at $0.2 \mathrm{mM} / \mathrm{kg}$. Typical acquisition entailed a phase sensitive inversion recovery technique with a spatial resolution $2.1 \times 2.1 \times 8 \mathrm{~mm}^{3}$. Post-processing was done using QMass 7.2 software (Medis, Leiden, The Netherlands). Infarct sizing was performed in both semi-quantitative (using a standard transmural categorization score ${ }^{15}$ of $1-4$, where $1=$ no infarct, $2=$ less than $25 \%$ transmural involvement, $3=25-50 \%, 4>50 \%$ ) and quantitative fashion using manual delineation in a slice-by-slice analysis with infarct tissue expressed in grams 16 .

\section{Randomization and Masking}

SCIPIO was performed in two sequential Stages (A and B). To assess the feasibility and immediate safety profile (i.e., short-term adverse effects) of CSC therapy, in Stage A nine consecutive patients were assigned to the treatment arm followed by assignment of four consecutive patients to the control arm (Fig. 1). Then, in Stage B, patients were randomized to the treated and control arms in a 2:3 ratio using an adaptive block randomization scheme and a block size of five (Fig. 1). Randomization was performed by two investigators (ARC and JHL) prior to final eligibility screening. Subject numbers were entered into a computer software program that randomly allocated treatment assignment using the aforementioned ratio. The purpose of adaptive block randomization was to attempt to correct the imbalance between the control and the treated groups, which stemmed from the fact that most eligible patients wished to be treated with CSCs. An open-label study design was adopted because blinding would have required a cardiac catheterization with placebo infusion in control subjects. However, the investigator who performed the echocardiographic analyses (MFS) was blinded to group assignment.

The study protocol was reviewed and approved by the Institutional Review Board for the University of Louisville. An independent Data and Safety Monitoring Board reviewed trial progress. Patients meeting initial eligibility criteria were approached before CABG surgery (within 2 weeks); prior to enrollment, all patients agreed to and signed an IRB-approved statement of informed consent. The study was performed in accordance with the principles of the Declaration of Helsinki. Trial investigators maintained compliance with the FDA regulations outlined in document 21 CFR 312, subpart D, regarding the stopping of the study and the procedures to be followed in the event of severe adverse events related to the administration of CSCs (Online Supplement).

Data are reported as means \pm SEM. Comparisons between two groups were performed with paired or unpaired Student's $t$ tests, as appropriate. 


\section{Role of the Funding Source}

The sponsors of the study had no role in study design, data collection, data analysis, data interpretation, or writing of the report. The corresponding author had full access to all of the data and had final responsibility for the decision to submit the article for publication.

\section{Results}

Investigational New Drug (IND) approval from the FDA was obtained on August 8, 2008. The study was initiated in February 2009. The first patient was enrolled on March 13, 2009 and received autologous CSCs on July 17, 2009. As of April 1, 2011, CSCs have been successfully isolated and expanded in 80 out of 81patients (the only failure was observed in a patient with cardiac amyloidosis). The numbers of patients screened, enrolled, and excluded are summarized in Fig. 1. Here we report the interim results obtained in 16 CSCtreated and 7 control patients.

Demographic data are summarized in Table 1. By design, all patients had at least one old MI; the average age of the infarct was $3.7 \pm 3.0$ years. In 5 of the 7 control and 15 of the 16 CSC-treated patients, there was evidence of a transmural MI (details are provided in the Online Supplement). Fifteen patients received $1 \times 10^{6} \mathrm{CSCs}$, whereas one received $0.5 \times$ $10^{6} \mathrm{CSCs}$; in this patient, LVEF increased by 16.7 units at 4 months after CSCs.

\section{CSCs}

C-kit ${ }^{+}$CSCs were characterized by immunolabeling, confocal microscopy, and FACS analysis (Fig. 2A; supplemental Fig. 2). The fraction of c-kit ${ }^{+}$cells varied from $75 \%$ to $98 \%$ (average, $88.0 \pm 1.7 \%$ ) (Fig. 2B). CSCs committed to the myocyte, SMC and EC lineages constituted $0.1 \%$ to $2.7 \%$ of the population $(1.1 \pm 0.2 \%$ ) (Fig. $2 \mathrm{~B})$. Telomere length, averaged $7.5 \mathrm{kbp}$ (range 6.8 to $8.1 \mathrm{kbp}$; Fig. 3A and supplemental Fig. 2B). These values were significantly higher than those associated with replicative senescence of human cells, i.e., $1.5-2 \mathrm{kbp}^{17}$. Additionally, telomerase activity was high in all CSC samples (Fig. 3B). The significant growth reserve of CSCs was confirmed by the measurement of PDT, which never exceeded $31 \mathrm{~h}$ (Fig. 3C). The well-preserved telomere-telomerase axis in CSCs was consistent with the relatively small percentage of CSCs positive for the senescenceassociated protein $\mathrm{p} 16^{\mathrm{INK} 4 \mathrm{a}}$ (supplemental Fig. 3), which prevents permanently the reentry of stem cells into the cell cycle ${ }^{18}$. Thus, CSCs expanded in culture retained a robust capacity for further cell division. There was no relation between age of the patients and either telomere length or telomerase activity (data not shown).

\section{Echocardiographic data}

Two CSC-treated patients could not be included in the echocardiographic analysis because of poor image quality (1 patient) and uncorrected aortic stenosis (1 patient). In the remaining 14 treated patients, LVEF, assessed by 3-D echocardiography, increased progressively from $30.3 \pm 1.9 \%$ before CSC infusion to $35.9 \pm 2.7 \% 1$ month later $(P=0.014)$ and $38.5 \pm 2.8 \%$ 4 months later $(P=0.001$, Fig. 4A). In the eight patients who have completed 1 year of follow-up, LVEF increased further from $39.2 \pm 3.6 \%$ at 4 months to $42.5 \pm 4.1 \%$ at 1 year (Fig. 4B); although this increase was not statistically significant $(P=0.159)$, it suggests that 
CSCs continue to improve LV function beyond the first 4 months. The net increase in LVEF from baseline was $8.2 \pm 2.0 \mathrm{EF}$ units at 4 months $(P=0.001)$ and $12.3 \pm 2.1$ at 12 months $(P=0.0007)$ (Fig. 4C). An improvement in LVEF was noted in 12 out of the 14 patients at 4 months (Fig. 4A) and 8 out of the 8 patients at 1 year (Fig. 4B).

The increase in LVEF was associated with an improvement in the regional wall motion score, both in the infused LV regions (from $1.97 \pm 0.13$ at baseline to $1.78 \pm 0.12$ at 4 months, $P=0.007$ ) and in all LV segments combined (from $1.91 \pm 0.09$ to $1.73 \pm 0.09$, $P=0.005$ ) (Figs. 4D and E).

In contrast, in the 7 control patients with 4 months of follow-up, none of these variables changed appreciably over the same time-interval; for example, LVEF averaged $30.1 \pm 2.4 \%$ at 4 months after $\mathrm{CABG}$ surgery and $30.2 \pm 2.5 \%$ at 8 months (Fig. $4 \mathrm{~A}$ ).

\section{cMR data}

cMR with gadolinium was performed in seven treated patients. Reasons for exclusion were ICD placement, eGFR $<40 \mathrm{ml} / \mathrm{min} / 1.73 \mathrm{~m}^{2}$, and non-CABG post-operative status with recently placed metal hardware. The infarct weight, which was $32.6 \pm 6.3 \mathrm{~g}$ before CSCs, decreased, on average, by $7.8 \pm 1.7 \mathrm{~g}$ at 4 months after CSC therapy $(P=0.004)$ and $9.8 \pm 3.5$ $\mathrm{g}$ at 12 months $(P=0.037)$ (Fig. 5). Hence, the relative decrease in infarct size was $24 \%$ and $30 \%$ at 4 and 12 months after CSC treatment, respectively. A reduction in infarct size was also shown by the semi-quantitative infarct score index (supplemental Fig 4). Measurements of $\mathrm{LV}$ wall thickening by $\mathrm{cMR}$ demonstrated a significant $(P=0.01)$ improvement at 4 months (supplemental Fig. 5), confirming the echocardiographic data.

\section{Functional status}

In the 16 treated patients, the NYHA functional class decreased from $2.19 \pm 0.16$ before CSC infusion to $1.63 \pm 0.164$ months later $(P=0.003)$ (Fig. 6A). Concomitantly, quality of life, as assessed by the MLHFQ score, improved markedly from $46.44 \pm 5.22$ to $26.69 \pm$ $4.92(P<0.0001)$ (Fig. 6C). In the 7 control patients, neither the NYHA class nor the MLHFQ score changed appreciably over the corresponding 4-month interval (2.0 vs. $1.71 \pm$ $0.18[P=0.172]$ and $38.14 \pm 10.53$ vs. $40.43 \pm 9.20[P=0.804]$, respectively) (Figs. 6A and C). In CSC-treated patients, the improvements in NYHA and MLHFQ score were even more pronounced at 1 year (Figs. 6B and D).

\section{Adverse effects}

No adverse effects ascribable to CSCs have been observed (Table 2). Specifically, none of the CSC-treated patients has experienced non-fatal MI (immediately after CSC infusion or during follow-up), death, tumor formation, ventricular arrhythmias, systemic infection, stroke, allergic reactions, or coronary revascularization. On the 24-h ambulatory ECG monitoring performed on 2 separate occasions ( 1 and 4 weeks after CSC therapy), no tachyarrhythmias were noted. One treated patient was hospitalized for HF, one treated and two control patients were hospitalized for angina, and one control underwent percutaneous coronary revascularization. In one treated patient, a dissection of the left internal mammary artery graft was produced during balloon inflation; this was repaired with a stent and there 
have been no complications over the ensuing 2 years. The information provided by SCIPIO regarding the safety and feasibility of using CSCs in humans is further described in the Online Supplement.

\section{Discussion}

Our results suggest that i) CSCs can be reproducibly isolated and expanded from $\sim 1 \mathrm{~g}$ of myocardial tissue harvested during cardiac surgery, ii) infusion of $1 \times 10^{6}$ autologous CSCs is not associated with apparent adverse effects for up to 1 year, and iii) infusion of autologous CSCs results in a striking improvement in LV systolic function, at 4 months later and even more pronounced 1 year after infusion and is associated with increased functional capacity, improved quality of life, and reduced LV scar size.

CSCs are particularly attractive for cardiovascular applications, because they normally reside in the adult heart and can be reproducibly isolated and expanded, even from endomyocardial biopsies ${ }^{12}$. These cells are thought to be responsible for replenishing the pool of cardiac myocytes and cardiac vascular cells that die during the life of the organism ${ }^{5-8}$.

The initial results of SCIPIO are consistent with this preclinical work and compare favorably with previous clinical studies using a variety of non-cardiac stem/progenitor cells in patients with ischemic cardiomyopathy ${ }^{4}$ (supplemental Table 2). In particular, the increase in LVEF observed in this study $(8.2 \pm 2.0 \%$ at 4 months and $12.3 \pm 2.1 \%$ at 1 year after CSC therapy) compares very favorably with prior studies of intracoronary bone marrow mononuclear cell infusion in a similar patient population (individuals with old MIs and impaired LV function), which have reported an increase in LVEF of $~ 3 \%$ (TOPCARE-CHD) ${ }^{20}, 7 \%$ (IACT study) ${ }^{21}, 6 \%$ (STAR-heart study) ${ }^{22}$, and $5 \%{ }^{23}$.

The optimal dose of CSCs remains to be determined. The dose of CSCs employed in SCIPIO $\left(1 \times 10^{6}\right)$ was selected conservatively on the basis of preclinical studies in pigs ${ }^{8}$ and of the growth characteristics of the cells. Our CSC population has an average telomere length of $7.5 \mathrm{kbp}$ (Fig. 3A). Because $130 \mathrm{bp}$ of telomeric DNA are lost after each division ${ }^{7}$ and replicative senescence occurs when telomeres reach $1.5-2 \mathrm{kbp}$, a single engrafted CSC could, in principle, divide 42 times prior to irreversible growth arrest, generating $4 \times 10^{12}$ cells. We have recently demonstrated that CSCs can be isolated and expanded from small $(\sim 5 \mathrm{mg})$ endomyocardial biopsies ${ }^{12}$. Therefore, in future studies it will be possible to examine the effects of larger numbers of autologous CSCs and in a much wider population of patients with HF using endomyocardial biopsies. Since CSCs can be frozen for subsequent use, it is conceivable that even better results may be obtained by repeated infusions in the same patient.

We elected to deliver CSCs at $4 \pm 1$ months after CABG surgery in order to allow myocardial stunning and/or hibernation ${ }^{24}$ to resolve and any improvement in LV function secondary to revascularization to occur. The appropriateness of this decision is supported by the high number of patients who were excluded because of an increase in LVEF during the first 4 months after CABG surgery (Fig. 1). It is generally agreed that, by 4 months after 
CABG, stunned and/or hibernating myocardium has recovered and LV function is relatively stable ${ }^{25-27}$. Indeed, no improvement was noted in the 7 control patients. Therefore, it is unlikely that the improvement in LV systolic performance noted herein in CSC-treated patients reflected the effects of CABG surgery.

The mechanism whereby CSCs produced their salubrious effects is unclear. The reduction in infarct size observed by cMR is consistent with cardiac regeneration, although it is uncertain whether regeneration, if it occurred, was mediated by differentiation of the injected CSCs, activation of resident CSCs, or both. Answering this critical question will necessitate the development of strategies to track the long-term fate of CSCs in patients. Other mechanisms (e.g., paracrine actions resulting in inhibition of apoptosis, inhibition of fibrosis, and/or enhanced contractile performance) cannot be excluded. Regardless of the mechanism, it is noteworthy that autologous CSCs improved LV performance despite the presence of mature scars that were, on average, $\sim 4$ years old.

Limitations of SCIPIO include the small number of patients and the absence of a placebotreated arm (which resulted in the open-label design). These features, which are common to many Phase I trials, reflect the novel nature of the therapy, and the fact that blinding was not possible because it would have necessitated intracoronary infusion of vehicle; nevertheless, all echocardiograms were analyzed without knowledge of treatment allocation. Any potential conditioning effect of the brief coronary occlusions in treated patients would be short-lived (48-72 h) ${ }^{24}$, and thus could not account for the long-term benefits observed. We emphasize that SCIPIO was designed to investigate the safety and feasibility of performing intracoronary CSC infusion in patients with severe HF, not to determine efficacy. All efficacy data need to be verified in larger studies.

In conclusion, the initial results of SCIPIO suggest that intracoronary infusion of autologous CSCs in patients with chronic ischemic cardiomyopathy and severe HF is feasible, safe, and apparently highly efficacious in restoring LV systolic function up to 1 year after treatment. These data warrant larger, Phase II studies of CSCs.

\section{Panel: Research in Context}

Systematic Review-A search for original publications was performed within the PubMed database using the terms "c-kit", "cardiac stem cell", "stem cell therapy", "bone marrow", "myocardial infarction" and "chronic ischemic heart failure". No studies of c-kit+ CSCs were identified in human subjects. All in vivo studies of CSCs were performed in animal models. Twelve clinical trials were found that utilized bone marrow-derived cells for the treatment of HF secondary to chronic ischemic cardiomyopathy (the setting relevant to SCIPIO) (supplemental Table 2). In four of these studies ${ }^{20-23}$, bone marrow cells were infused intracoronarily (in one of them ${ }^{23}$, they were infused during CABG surgery), in three studies cells were injected transendocardially, and in five they were injected epicardially during CABG surgery (supplemental Table 2).

Interpretation-The present study is the first report of CSC therapy in humans. It is a significant addition to the current literature because it introduces a new potential treatment for HF. This work represents the clinical translation of a large body of preclinical studies 
demonstrating salubrious effects of CSCs on LV function and structure in mouse, rat, dog, and pig models of post-MI HF ${ }^{29}$. SCIPIO differs from all previous trials of cell therapy for heart disease, which have used non-cardiac cell products (mostly bone marrow-derived cells ${ }^{4}$ ). The overwhelming majority of these previous studies were conducted in patients with acute MI; relatively few sought to treat HF caused by chronic ischemic cardiomyopathy, which was the setting for SCIPIO. Our present results indicate an increase in global LV function (LVEF) of $8 \mathrm{EF}$ units at 4 months and $12 \mathrm{EF}$ units at 12 months after CSC administration. Among the previous studies of bone marrow cells in chronic ischemic cardiomyopathy (supplemental Table 2), the four that used intracoronary infusion reported increases in LVEF of $3 \mathrm{EF}$ units (TOPCARE-CHD) ${ }^{20}, 7$ units (IACT) ${ }^{21}$, and 6 units (STAR-heart) ${ }^{22}$ at 3 months, 5 units ${ }^{23}$ at 6 months, and 6 units (STAR-heart) at 1 and 5 years. Of the three studies of transendocardial injection, one reported an increase in LVEF of 5.5 units at 2 months, and two reported no significant improvement at 6 months and 1 year (supplemental Table 2). Epicardial cell delivery differs profoundly from intracoronary infusion in that it requires a thoracotomy. Thus, while the primary purpose of our phase I trial was to assess the safety and feasibility of using this distinct and unique population of cells, the treatment effects observed are very encouraging and compare favorably with previous trials of bone marrow cells. The present results provide a strong rationale for further studies of CSC therapy in patients with severe HF secondary to ischemic cardiomyopathy, who currently have a poor prognosis.

\section{Supplementary Material}

Refer to Web version on PubMed Central for supplementary material.

\section{Acknowledgments}

Funding. Supported by the University of Louisville Research Foundation and NIH Grant R37HL081737.

This study was supported by the University of Louisville Research Foundation and NIH Grant R37HL081737.

\section{References}

1. Coronary Heart Disease Statistics. British Heart Foundation; 2003.

2. Roger VL, Go AS, Lloyd-Jones DM, et al. Heart disease and stroke statistics-2011 update: a report from the American Heart Association. Circulation. 2011; 123(4):e18-e209. [PubMed: 21160056]

3. Stewart S, MacIntyre K, Hole DJ, Capewell S, McMurray JJV. More malignant than cancer? Five year hospitalization after a first hospitalization with heart failure. Eur Heart Fail. 2001; 3:315-22.

4. Abdel-Latif A, Bolli R, Tleyjeh IM, et al. Adult bone marrow-derived cells for cardiac repair: a systematic review and meta-analysis. Arch intern Med. 2007; 167(10):989-97. [PubMed: 17533201]

5. Beltrami AP, Barlucchi L, Torella D, et al. Adult cardiac stem cells are multipotent and support myocardial regeneration. Cell. 2003; 114(6):763-76. [PubMed: 14505575]

6. Linke A, Muller P, Nurzynska D, et al. Stem cells in the dog heart are self-renewing, clonogenic, and multipotent and regenerate infarcted myocardium, improving cardiac function. Proc Natl Acad Sci U S A. 2005; 102(25):8966-71. [PubMed: 15951423]

7. Bearzi C, Rota M, Hosoda T, et al. Human cardiac stem cells. Proc Natl Acad Sci U S A. 2007; 104(35):14068-73. [PubMed: 17709737]

8. Bolli R, Jneid H, Tang XL, et al. Intracoronary administration of cardiac stem cells improves cardiac function in pigs with old infarction. Circulation. 2006; 114(18 Supplement):II_239. 
9. Dawn B, Stein AB, Urbanek K, et al. Cardiac stem cells delivered intravascularly traverse the vessel barrier, regenerate infracted myocardium, and improve cardiac function. Proc Natl Acad Sci U S A. 2005; 102(10):3766-71. [PubMed: 15734798]

10. Tang XL, Rokosh G, Sanganalmath SK, et al. Intracoronary administration of cardiac progenitor cells alleviates left ventricular dysfunction in rats with a 30-day-old infarction. Circulation. 2010; 121(2):293-305. [PubMed: 20048209]

11. Fischer KM, Cottage CT, Wu W, et al. Enhancement of myocardial regeneration through genetic engineering of cardiac progenitor cells expressing Pim-1 kinase. Circulation. 2009; 120(21):207787. [PubMed: 19901187]

12. D'Amario D, Fiorini C, Campbell PM, et al. Functionally competent cardiac stem cells can be isolated from endomyocardial biopsies of patients with advanced cardiomyopathies. Circ Res. 2011; 108(7):857-61. [PubMed: 21330601]

13. D'Amario D, Cabral-Da-Silva MC, Zheng H, et al. Insulin-like growth factor-1 receptor identifies a pool of human cardiac stem cells with superior therapeutic potential for myocardial regeneration. Circ Res. 2011; 108(12):1467-81. [PubMed: 21546606]

14. Schiller NB, Shah PM, Crawford M, et al. Recommendations for quantitation of the left ventricle by two-dimensional echocardiography. American Society of Echocardiography Committee on Standards, Subcommittee on Quantitiation of Two-Dimensional Echocardiograms. J Am Soc Echocardiogr. 1989; 2(5):358-67. [PubMed: 2698218]

15. Kim RJ, Shah DJ, Judd RM. How we perform delayed enhancement imaging. J Cardiovasc Magn Reson. 2003; 5(3):505-14. [PubMed: 12882082]

16. Flett AS, Hasleton C, Cook C, et al. Evaluation of techniques for the quantification of myocardial scar of differing etiology using cardiac magnetic resonance. JACC Cardiovasc Imaging. 2011; 4(2):150-6. [PubMed: 21329899]

17. Aubert G, Lansdorp PM. Telomeres and aging. Physiol Rev. 2008; 88(2):557-79. [PubMed: 18391173]

18. Beausejour CM, Campisi J. Ageing: balancing regeneration and cancer. Nature. 2006; 443(7110): 404-5. [PubMed: 16957734]

19. Rota M, Padin-Iruegas ME, Misao Y, et al. Local activation or implantation of cardiac progenitor cells rescues scarred infarcted myocardium improving cardiac function. Circ Res. 2008; 103(1): 107-16. [PubMed: 18556576]

20. Assmus B, Honold J, Schachinger V, et al. Transcoronary transplantation of proenitor cells after myocardial infarction. N Engl J Med. 2006; 355(12):1222-32. [PubMed: 16990385]

21. Strauer BE, Brehm M, Zeus T, et al. Regeneration of human infarcted heart muscle by intracoronary autologous bone marrow cell transplantation in chronic coronary artery disease: the IACT Study. J Am Coll Cardiol. 2005; 46(9):1651-8. [PubMed: 16256864]

22. Strauer BE, Youssef M, Schannwell CM. The acute and long-term effects of intracoronary stem cell transplantation in 191 patients with chronic heart failure: the STAR-heart study. Eur J Heart Fail. 2010; 12:721-729. [PubMed: 20576835]

23. Hu S, Liu S, Zheng Z, et al. Isolated coronary artery bypass graft combined with bone marrow mononuclear cells delivered through a graft vessel for patients with previous myocardial infarction and chronic heart failure. J Am Coll cardiol. 2011; 57(24):2409-15. [PubMed: 21658561]

24. Heusch G. Hibernating myocardium. Physiol Rev. 1998; 78(4):1055-85. [PubMed: 9790569]

25. Vanoverschelde JL, Depre C, Gerber BL, et al. Time course of functional recovery after coronary artery bypass graft surgery in patients with chronic left ventricular ischemic dysfunction. Am $\mathrm{J}$ Cardiol. 2000; 85(12):1432-9. [PubMed: 10856388]

26. Lorusso R, La Canna G, Ceconi C, et al. Long-term results of coronary artery bypass grafting procedure in the presence of left ventricular dysfunction and hibernating myocardium. Eur $\mathbf{J}$ Cardiothorac Surg. 2001; 20(5):937-48. [PubMed: 11675178]

27. Mintz LJ, Ingels Nb Jr, Daughters GT 2nd, Stinson EB, Adlerman El. Sequential studies of left ventricular function and wall motion after coronary arterial bypass surgery. Am J Cardiol. 1980; 45(2):210-16. [PubMed: 6965556]

28. Malliaras K, Marban E. Cardiac cell therapy: where we've been, where we are, and where we should be headed. Br Med Bull. 2011; 98:161-85. [PubMed: 21652595] 
29. Chamuleau SAJ, Vrijsen KR, Rokosh DG, Tang XL, Piek JJ, Bolli R. Cell therapy for ischaemic heart disease: focus on the role of resident cardiac stem cells. Neth heart J. 2009; 17(5):199-207. [PubMed: 19484156] 


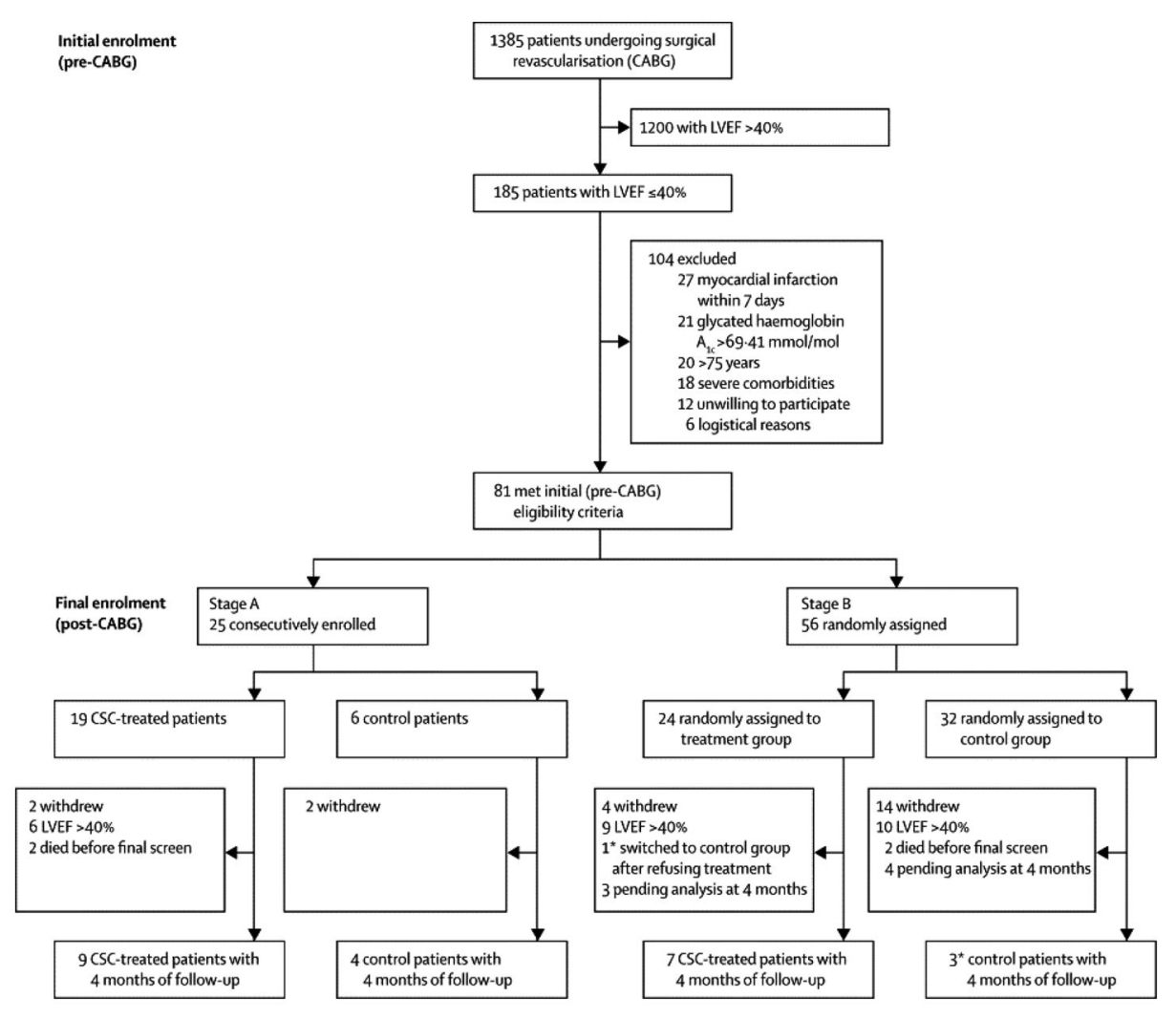

Figure 1.

Trial profile showing a summary of screening, exclusions, and enrollment. Patients were screened at two time points: during the 2 weeks preceding CABG surgery (initial eligibility screening and enrollment), and at $4 \pm 1$ months after CABG surgery (final enrollment). The trial consisted of two sequential Stages, A (non-randomized) and B (randomized). To assess feasibility and short-term safety of CSC therapy, in Stage A nine consecutive patients were assigned to the treatment arm followed by assignment of four consecutive patients to the control arm. Then, in Stage B, patients were randomized to the treated and control arms in a $2: 3$ ratio using a block randomization scheme and a block size of five. The Figure summarizes enrollment as of April 1, 2011. 

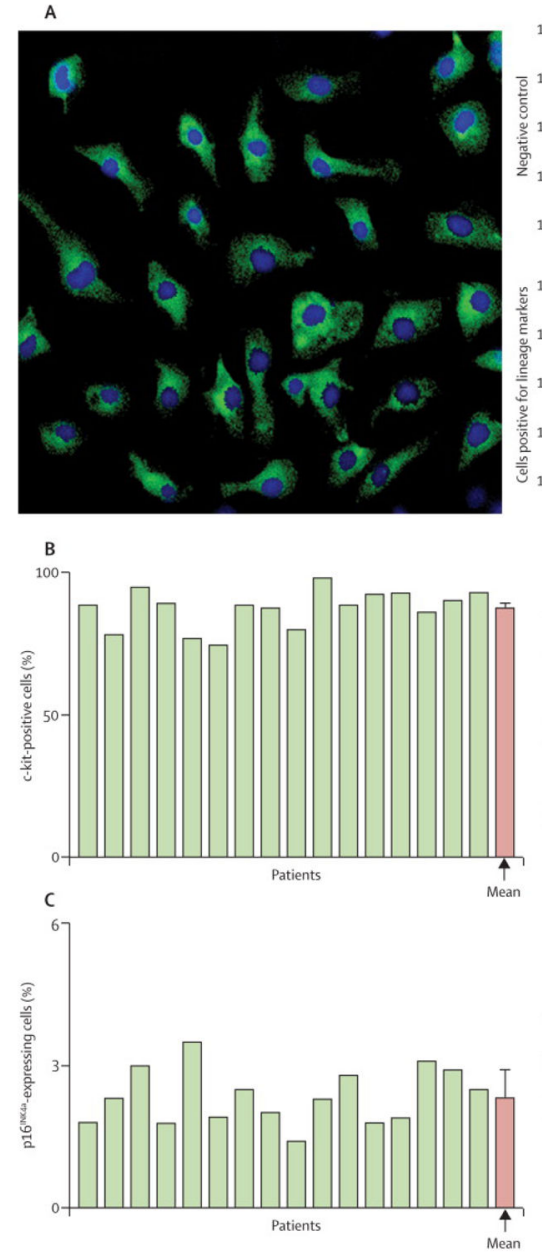
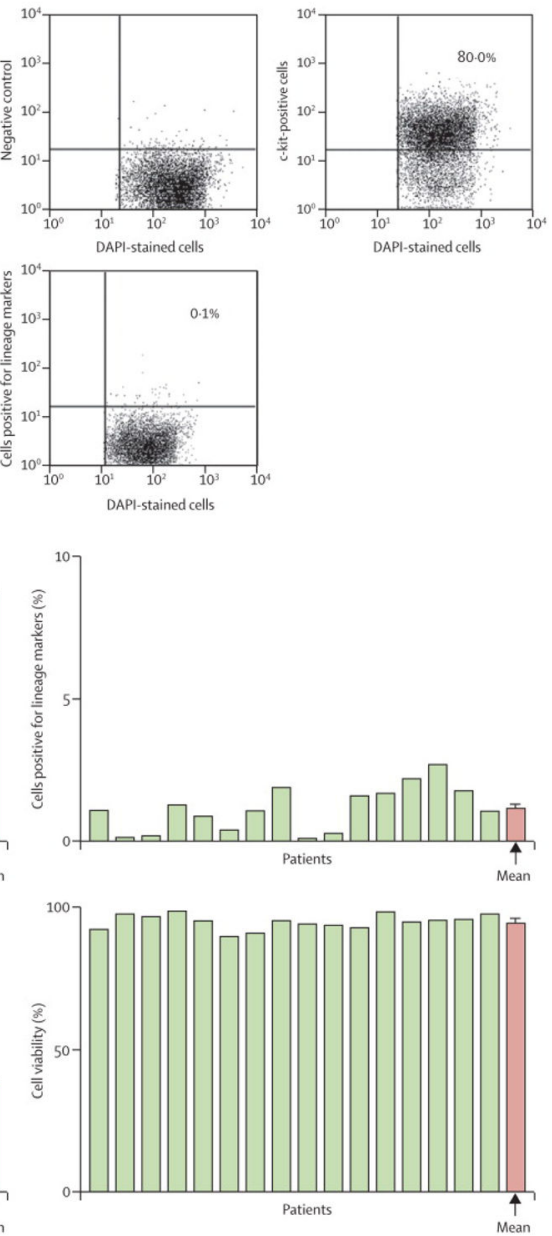

Figure 2.

Phenotype of CSCs prior to intracoronary delivery. A, confocal image illustrating the localization of c-kit (green) in CSCs and the FACS analysis of c-kit expression and lineage markers of CSCs for Subject 019. Numerical values are indicated. B, percentage of cells expressing c-kit, lineage markers of cardiac commitment, and the senescence-associated protein $\mathrm{p} 16^{\mathrm{INK} 4 \mathrm{a}}$. The percentage of viable cells in the final preparation is also illustrated. Yellow bars indicate individual values, red bars indicate means \pm SEM. 

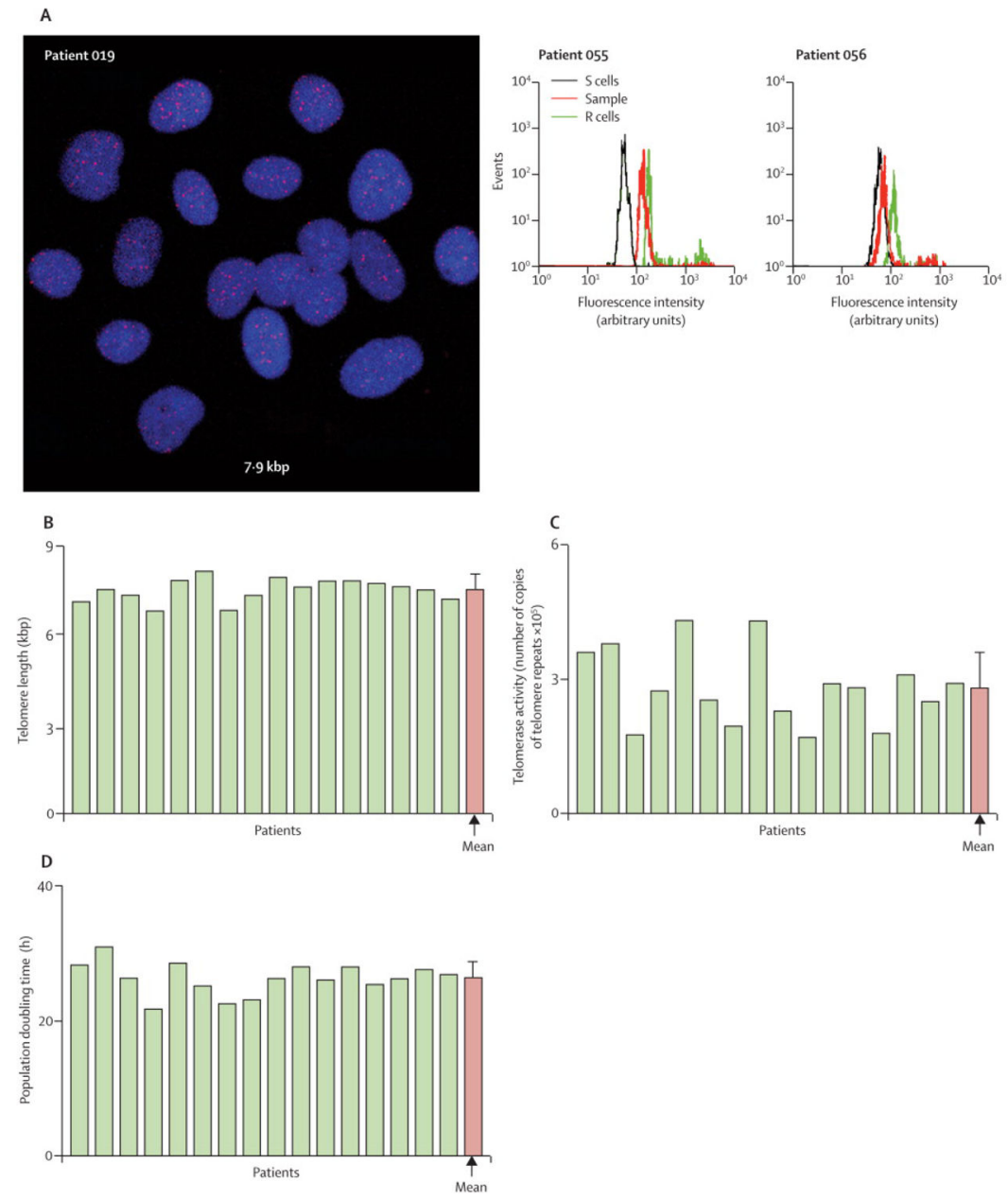

Figure 3.

Growth properties of CSCs prior to intracoronary delivery. A, telomeres in CSC nuclei (red dots) are identified by Q-FISH (left panel, Subject 019) and flow-FISH (central and right panels, Subjects 055 and 056). R cells with long telomeres (48 kbp) and S cells with short telomeres $(7 \mathrm{kbp})$ were used to compute absolute values. In each panel, telomere length is indicated. By flow-FISH, the histograms represent the intensity of PNA probe binding in gated CSCs (red) and control cells (green). B, telomerase activity in CSC lysates from each patient was detected by quantitative PCR. C, population doubling time in CSCs. Yellow bars indicate individual values, red bars indicate means \pm SEM. 

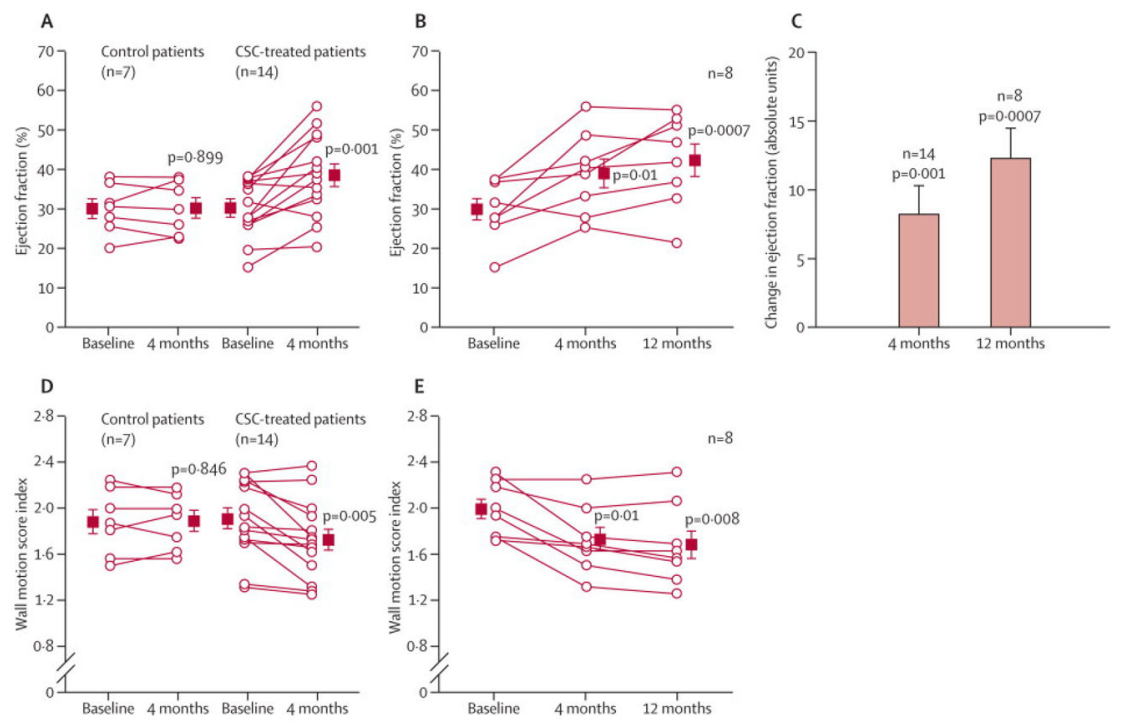

Figure 4.

Echocardiographic analysis at 4 and 12 months after baseline (baseline was $4 \pm 1$ month after surgical revascularization). A, left ventricular ejection fraction (EF) (measured by 3D echocardiography) at 4 months after baseline in control and treated patients. B, EF at 4 and 12 months after baseline in the eight treated patients that have 1 year of follow-up. $\mathbf{C}$, change in EF from baseline at 4 and 12 months in treated patients (absolute EF units). D, wall motion score index (WMSI) at 4 months after baseline in control and treated patients. E, WMSI at 4 and 12 months after baseline in the eight treated patients that have 1 year of follow-up. Data are means \pm SEM. 

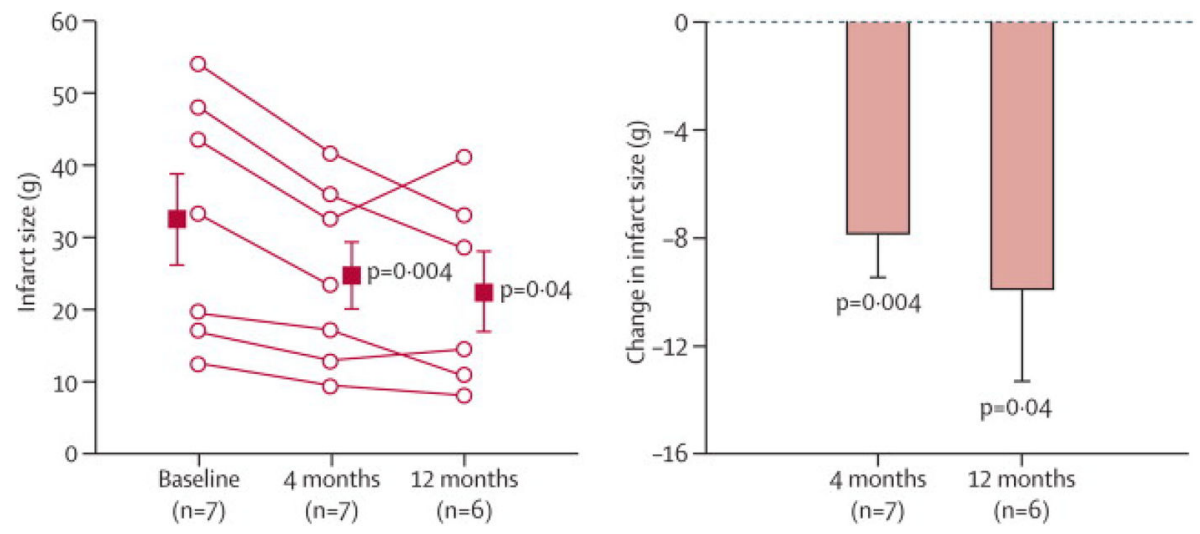

Figure 5.

Cardiac magnetic resonance (cMR) analysis at 4 and 12 months after baseline in treated patients (baseline was $4 \pm 1$ month after surgical revascularization). A, infarct size. B, change in infarct size from baseline. Data are means \pm SEM. 


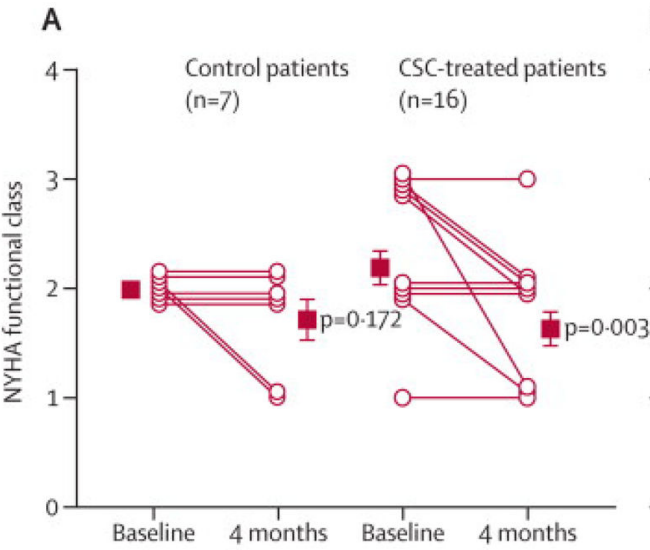

B

\section{C}

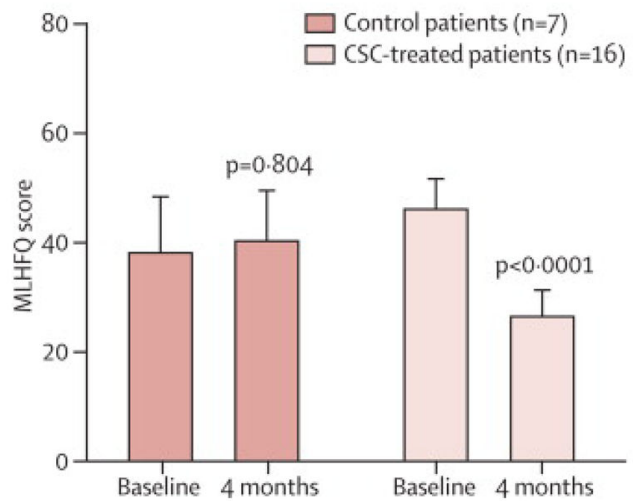

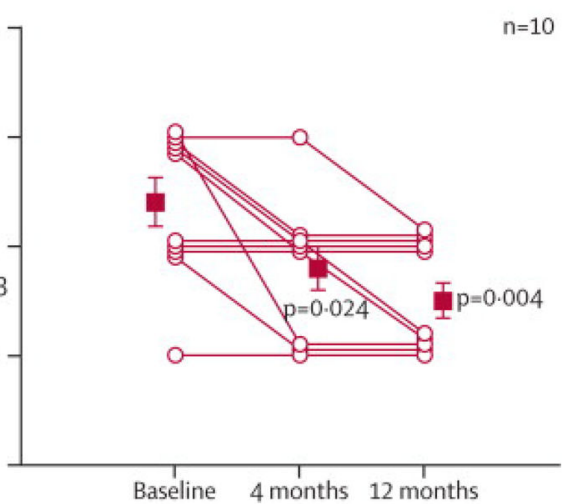

D

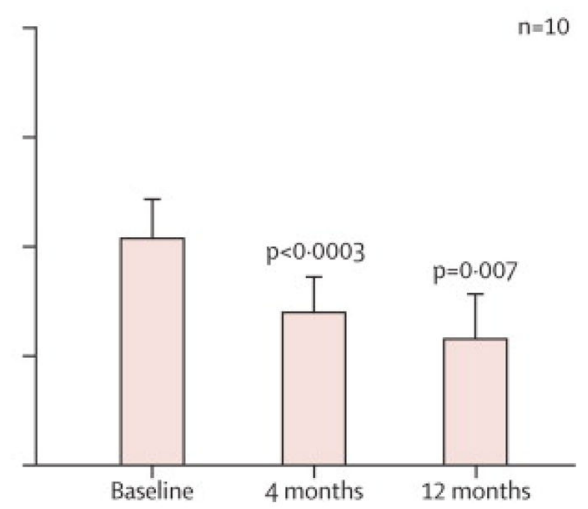

Figure 6.

Functional status/quality of life. New York Heart Association (NYHA) functional class (A and B) and quality of life (evaluated by the Minnesota Living with Heart Failure Questionnaire [MLHFQ] score) (C and D) at 4 and 12 months after baseline (baseline was 4 \pm 1 month after surgical revascularization). A, NYHA functional class and C, MLHFQ score at 4 months after baseline in control and treated patients. B, NYHA functional class and D, MLHFQ score at 4 and 12 months after baseline in the 10 treated patients that have 1 year of follow-up. Data are means \pm SEM. 

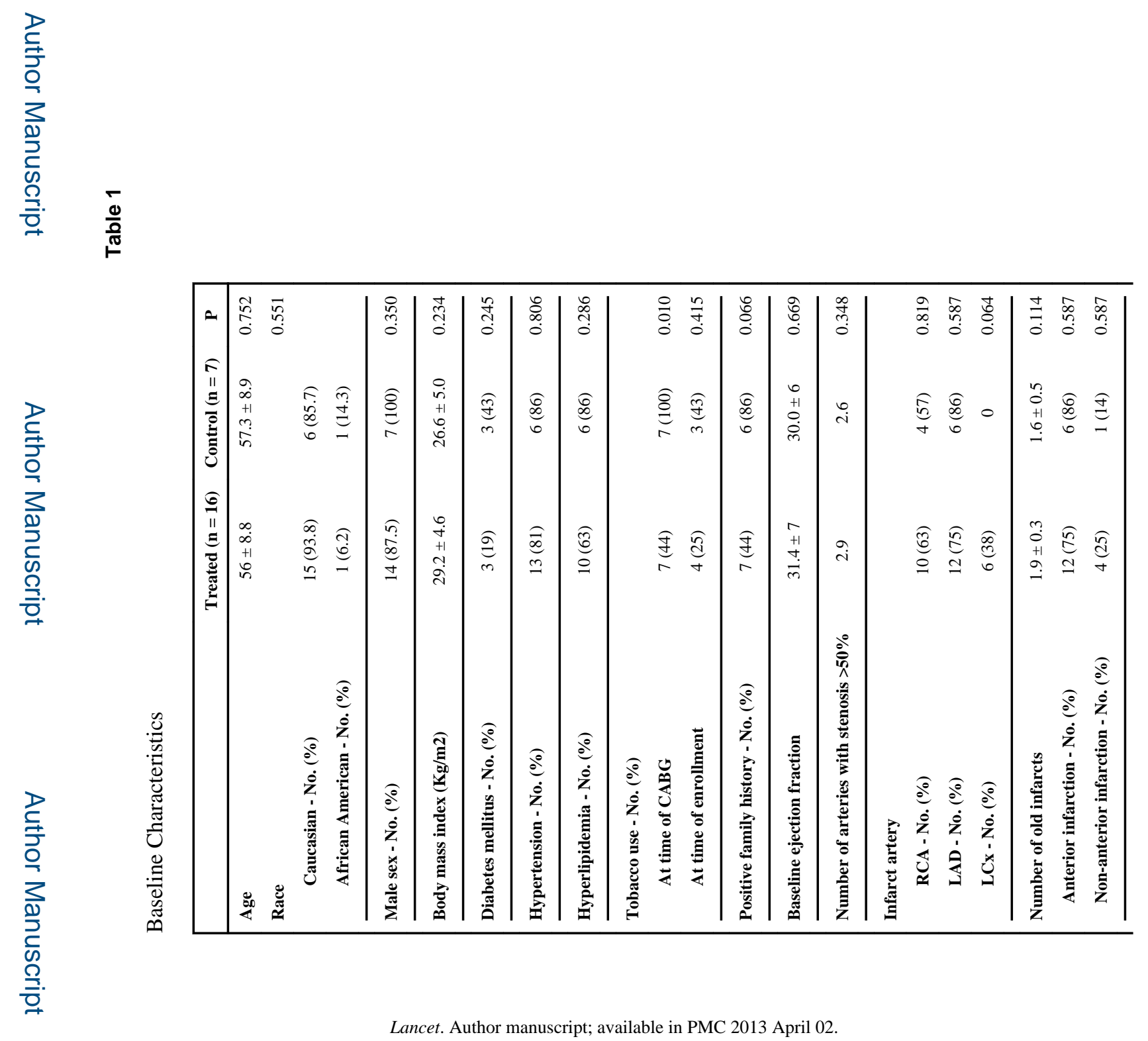


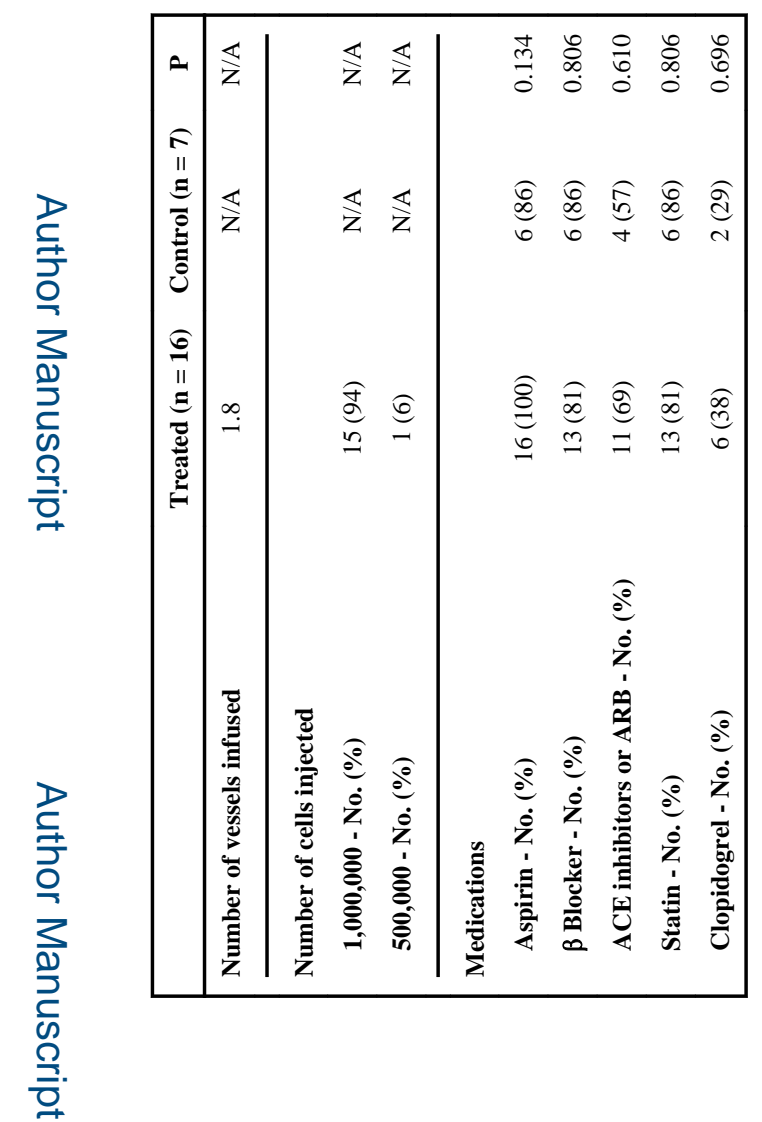

Lancet. Author manuscript; available in PMC 2013 April 02. 


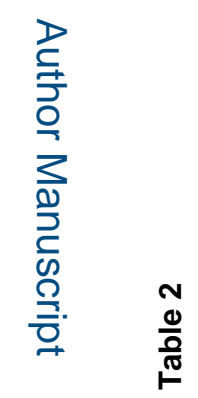

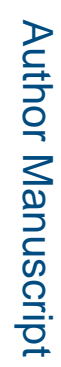
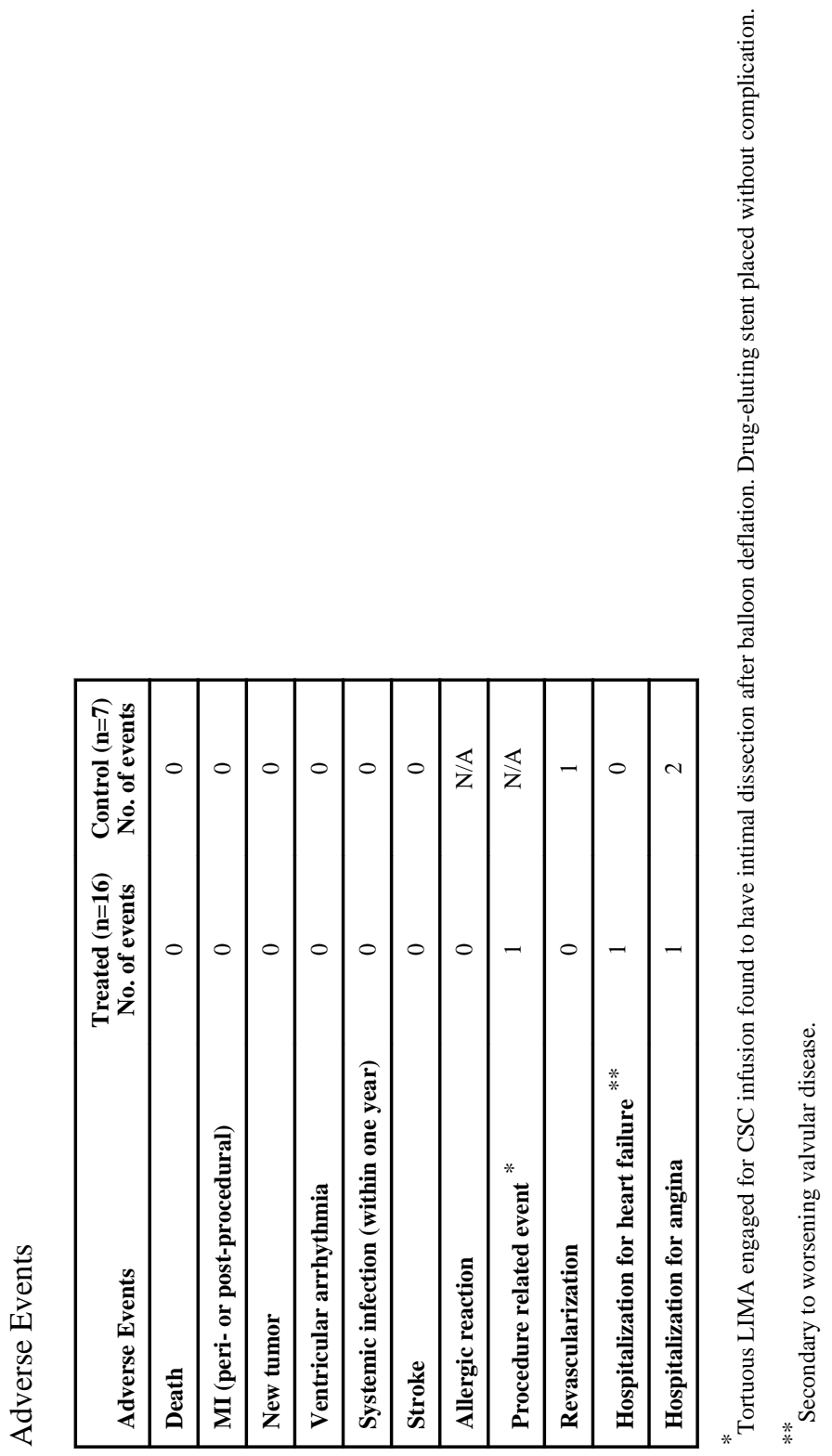

Lancet. Author manuscript; available in PMC 2013 April 02. 